\section{Disappearing roots. A case of widespread apical root resorption}

\author{
A. Omer, ${ }_{1}^{1}$ S. Abela, ${ }_{1}^{2}$ G. Gray, ${ }_{1}^{3}$ N. Atack, ${ }_{1}^{4}$ and P. Crawford ${ }^{5}$
}

IN BRIEF
Reviews some of the factors associated
with root resorption.
The widespread resorption seen in this
case suggests an underlying condition.
Periodic radiographs are essential in
patients with a history of multiple root
resorption.
Non-setting calcium hydroxide has been
proven to be an effective intra-canal
medicament in the treatment of root
resorption of an inflammatory origin.

This article describes the case of widespread apical root resorption of unknown origin in a 16-year-old male. Radiographic investigations showed widespread root resorption affecting permanent molars, premolars and incisors to a varying degree. Haematological and endocrine investigations were all within normal ranges. The mechanisms for the findings are considered.

\section{INTRODUCTION}

Resorption is the process which results in the loss of tooth tissue with dentine, cementum or alveolar bone all being involved in equal amounts. Based on the site of origin, root resorption may be classified as either internal or external. ${ }^{1}$

External resorption can be classified based on the clinical and histological findings to four different types:?

1) external surface resorption

2) external inflammatory root resorption

3) ankylosis

4) replacement resorption.

It is often difficult to identify a specific cause for resorption, because root resorption is usually asymptomatic. The aetiology is often multi-factorial unless a specific cause can be identified. This is further emphasised by Andreason's work ${ }^{2}$ for which he identified four factors that could contribute equally to the development of inflammatory resorption: 1) Injury to the periodontal ligament, 2) Initiation of surface resorption, 3) Communication with necrotic pulp tissue, 4) carious lesions and patent dentinal tubules.

\footnotetext{
$1^{*-5}$ Division of Child Dental Health, Bristol Dental Hospital, Lower Maudlin Street, Bristol, BS1 2LY

${ }^{*}$ Correspondence to: Dr Ahmed Omer

Email: doctorahmed@gmail.com
}

\section{Refereed Paper}

Accepted 23 March 2009

DOI: $10.1038 /$ sj.bdj.2009.414

${ }^{\circledR}$ British Dental Journal 2009; 206: 525-527

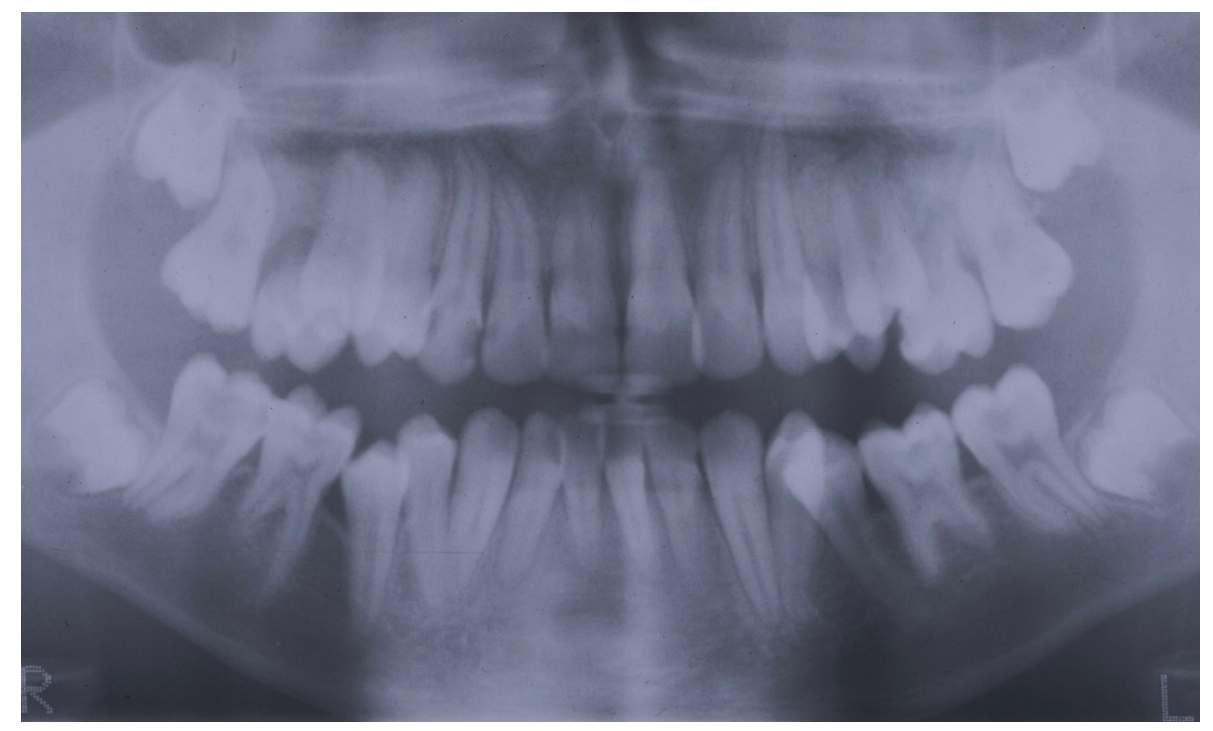

Fig. 1 DPT at the age of 13 years showing external apical root resorption of the four first permanent molar teeth and the third molar teeth impacting against the second molars

This report illustrates a case of external apical resorption involving teeth with no associated abnormalities.

\section{CASE REPORT}

A 16-year-old Caucasian male was referred by his general dental practitioner who had noticed radiographic evidence of external resorption affecting multiple teeth. The patient was in good health with no significant past or present medical history and claimed to have no history of accidental dental trauma. The patient had not undergone orthodontic treatment in the past.

The referring practitioner had commenced root canal therapy by removing the necrotic remnants of pulpal tissue from the extensively carious maxillary right canine and lateral, as these teeth had given symptoms of discomfort.

On examination, there were no significant extra-oral findings. However, intra-oral examination revealed a heavily restored dentition and the patient's oral hygiene was very poor with widespread caries affecting many teeth.

A dental-panoramic tomogram (DPT) previously taken at the age of 13 years and three months revealed apical root resorption of the four first permanent molar teeth. These teeth were extracted a few months later (Fig. 1).

A more recent DPT (Fig. 2) at 16 years and two months of age demonstrated significant generalised root resorption. There was substantial resorption of the 


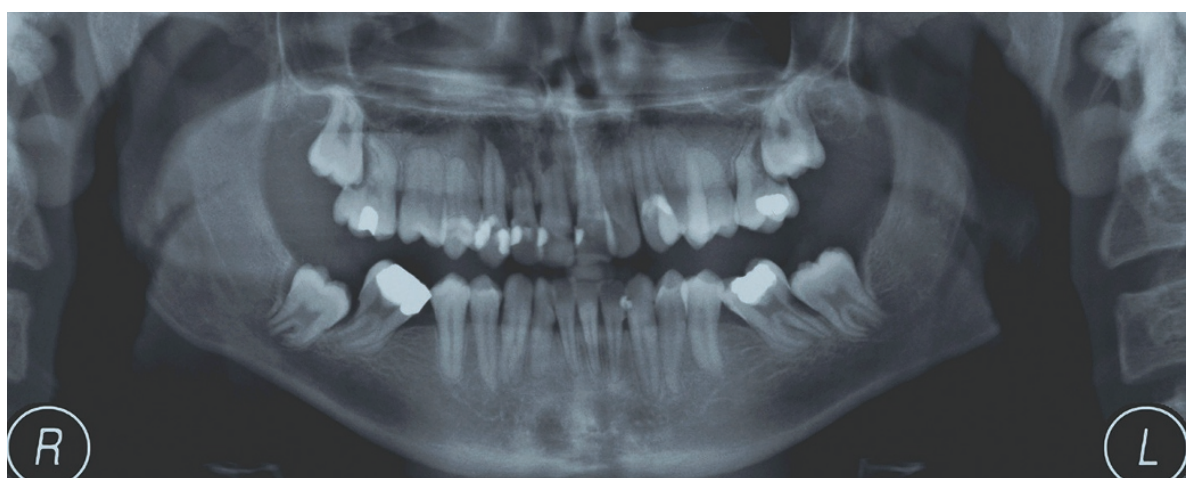

Fig. 2 DPT at the age of 16 years and two months showing multiple resorption

distal root of the maxillary and mandibular second permanent molars $(17,27,37$ and 47) (Fig. 3).

In addition, most of the premolar teeth (15, 14, 25, 35 and 45) showed blunted root apices with loss of approximately 2-3 $\mathrm{mm}$ of the apical root structures. The upper right lateral and canine teeth showed very severe apical resorption.

There was no evidence of bony pathology of these sites. DPT also revealed generalised moderate to severe horizontal bone loss; however, there was no evidence of periradicular periodontitis.

There was evidence of apical resorption affecting 11, 12 and 13 with associated bony pathology confirmed by intra-oral radiographs (Fig. 4). All teeth responded positively to vitality testing except for teeth 12 and 13 which were undergoing endodontic therapy.

A battery of haematological investigations and urinanalysis, testing for serum calcium, alkaline phosphatase, vitamin D, parathyroid hormone, osteocalcin, full blood count, urea and electrolytes, serum creatinine, liver function tests and thyroid function tests showed no endocrinological abnormalities nor any raised markers of inflammation.

The provisional diagnoses were made of:

1) Resorption associated with eruption and impaction for teeth 37 and 47

2) Resorption associated with dentine and pulpal infection for teeth 13 , 12 and 11

3) Idiopathic resorption for teeth 16, $15,14,24,25,26,34,35,36,46$, 45 and 44.

The treatment plan was to continue with the root canal therapy already commenced in teeth 12 and 13 by his general dental practitioner. In addition, non-setting calcium hydroxide paste was to be used as an intracanal medication and was to be changed at regular three months intervals, in an attempt to arrest the inflammatory resorption.

Recently, at the age of 17 years, a periapical radiograph taken by his dentist showed extensive apical root resorption to the 11 and 12 (Fig. 5). The dentist commenced root canal treatment on 11 and dressed teeth 11, 12 and 13 with non-setting calcium hydroxide (UltraCal, Ultradent Products, USA) and is to keep the patient under regular review.

This treatment is failing to control the resorption of the affected teeth. In the long term, if the anterior teeth need to be removed then a transitional acrylic removable prosthesis will be provided for the patient until a permanent prosthesis is a more realistic form of treatment.

\section{DISCUSSION}

Although the mechanism of dental resorption is believed to be very similar to that of bone resorption, the aetiology has not been fully elucidated.

Localised resorption of the teeth is commonly seen in response to pressure, as in the impaction of an erupting adjacent tooth, as a result of orthodontic treatment, as a result of exuberant demolition and repair following dental injuries - but infection and tumours can also be causes. ${ }^{3}$

However, generalised resorption is usually correlated with periodontitis, systemic illness and endocrine disturbances, which include: hyperphosphatemia; PapillonLefevre syndrome; Paget's disease; hypoparathyroidism; hypophosphatemia; hyperpatathyroidism; Gaucher disease; Turner syndrome; anachoresis; Goltz syndrome; hypophosphatasia. ${ }^{4}$

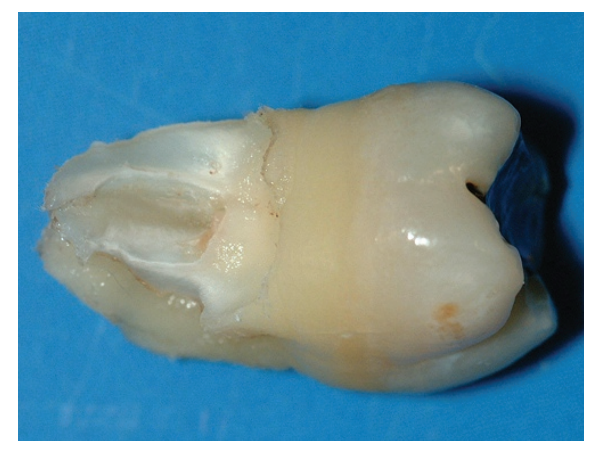

Fig. 3 Extracted lower right second molar showing severe resorption of the distal root

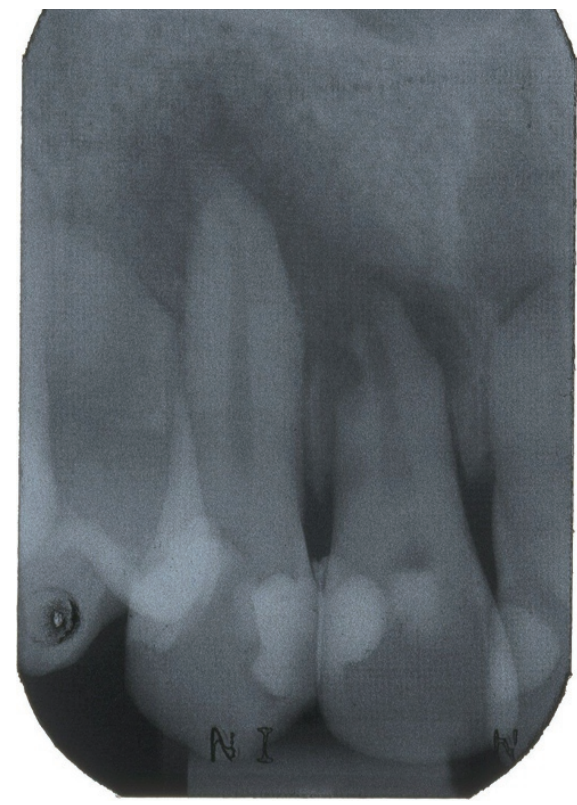

Fig. 4 Periapical radiograph at the age of 16 years and two months showing external inflammatory resorption of teeth 11 , 12 and 13

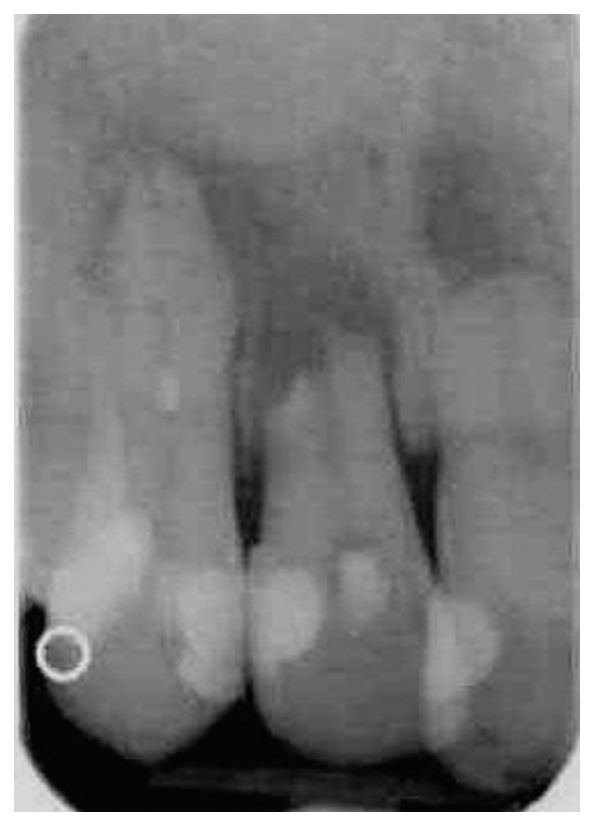

Fig. 5 Periapical radiograph at the age of 17 years showing extensive apical resorption of teeth 11 and 12 and arrested resorption in tooth 13 
Extensive investigation by colleague endocrinologist has so far failed to identify any significant abnormality. There was also no family history of root resorption. In addition, in the literature any tooth resorption without a known aetiology can be named as idiopathic root resorption. ${ }^{5}$ However, it is difficult to establish which of the factors the major determinant was as most probably multiple factors would have worked synergistically. ${ }^{6}$ In this case bacterial invasion through carious lesions and patent dentinal tubules may have resulted in chronic inflammation of the pulp as suggested by Andreason in 1984. ${ }^{2}$

Progressive resorption of teeth has previously been reported in patients with periodontal disease and poor oral hygiene. This has been attributed to inflammation of the collagen in the periodontal ligament. ${ }^{7}$ It is believed that Sharpey's fibres arising from the cementum are closely-packed when compared to those that are inserted into the alveolar bone. This makes cementum more resistant to resorption, and the loss of these closely-packed fibres, due to poor periodontal health, makes cementum less resistant to osteoclastic activity. In this case, the patient had poor plaque control and periodontal conditions, and the GDP is managing this issue for the patient.

Root resorption arising from impaction of molar teeth mainly affects destruction of the second primary molars by the influence of the first permanent molars or of the second permanent molars by the influence of the third permanent molars. Patients should be advised about the possible consequences of leaving impacted third molars in place; the DPT taken at the age of 13 years showed signs of third molar impaction and preventive extraction of the third molars at that age may have saved the second molar teeth. ${ }^{8}$

By contrast to the above, generalised external or internal resorption of teeth is not a commonly reported condition. The literature suggests that similar cases were asymptomatic and were discovered coincidentally on radiographic examination. ${ }^{9}$

Treatment of idiopathic root resorption has varied from extraction to endodontic treatment or watchful waiting. Rarely, calcitonin has been recommended as an interim root canal medicament to assist in the inhibition of oestoclastic bone and tooth resorption. The clinical value of calcitonin has yet to be evaluated, but it has been suggested that the material penetrates the dentinal tubules in an outward direction to have a direct effect externally on the resorption. ${ }^{10}$

The obturation of resorped teeth represents technical challenges due to abnormal apical foramina. This may be overcome by ensuring that the resorptive process has ceased and utilising a combination of techniques of cold and hot thermoplasticised gutta-percha. The following options can be considered as treatment in these circumstances:

1) Lateral condensation technique used for the apical third of the root to prevent any extrusion of the obturating material followed by the use of warm gutta-percha to seal the rest of the canal ${ }^{11}$

2) Anterograde seal of the apical part of the canal using Mineral trioxide aggregate (MTA) and standard techniques for the rest of the canal.

In the absence of any clear diagnosis in this case, the outcome for this patient's dentition must be poor. Local causes can be suggested for the resorption of individual sites in this patient's dentition such as infection in the anterior teeth and impaction in the molars. However, the widespread distribution of the resorptive process slightly suggests an underlying cause, as yet undiscovered or this may be due to genetic factors as described by Al-Qawasmi et al. ${ }^{12}$

This case illustrates the importance of regular clinical and radiographic follow up in such an eventuality.

The authors are grateful for the help of many colleagues in the investigation of this patient's case; most notably $\mathrm{Dr}$ M. J. T. Seymour and Ms E. K. Tyrrell, to whom they extend their thanks and appreciation.

1. Tronstad L. Root resorption - etiology, terminology and clinical manifestations. Endod Dent Traumatol 1988; 4: 241-252.

2. Andreasen J. External root resorption: its implication in dental traumatology, paedodontics, periodontics, orthodontics and endodontics. Int Endod J 1985; 18: 109-118.

3. Moazami F, Karami B. Multiple idiopathic apical root resorption: a case report. Int Endod J 2007; 40: 573-578.

4. Tangney N J. Hypophosphatasia: a case report and literature review. Ir Med J 1979; 72: 530-531.

5. Belanger G, Coke J. Idiopathic external root resorption of the entire permanent dentition: report of case. ASDC J Dent Child 1985; 52: 359-363.

6. Bakland L. Root resorption. Dent Clin North Am 1992; 36: 491-507.

7. Shafer W G, Levy B M. Textbook of oral pathology, 4th ed. Philadelphia: WB Saunders, 1983.

8. Nemcovsky C, Tal H, Pitaru S. Effect of non-erupted third molars on roots of approximal teeth. A radiographic, clinical and histologic study. J Oral Pathol Med 1997; 26: 464-469.

9. Liang H, Burkes E, Frederiksen N. Multiple idiopathic cervical root resorption: systematic review and report of four cases. Dentomaxillofac Radiol 2003; 32: 150-155.

10. Pierce A, Berg J, Lindskog S. Calcitonin as an alternative therapy in the treatment of root resorption. J Endod 1988; 14: 459-464.

11. Kinomoto $Y$, Noro T, Ebisu S. Internal root resorption associated with inadequate caries removal and orthodontic therapy. J Endod 2002; 28: 405-407.

12. Al-Qawasmi R, Hartsfield J J, Everett E, Flury L et al. Genetic predisposition to external apical root resorption. Am J Orthod Dentofacial Orthop 2003; 123: $242-252$ 